\section{Thorakoskopiekurs in Halle/Saale mit neuer Strategie der praktischen Ausbildung}

Vom 18. bis 20. Oktober 2018 fand in Halle/Saale der jährliche Thorakoskopiekurs unter der Schirmherrschaft der Sektion Endoskopie der Deutschen Gesellschaft für Pneumologie und Beatmungsmedizin statt.

Bei diesem Kurs wurden neben historischen Aspekten der Methode die Grundlagen hinsichtlich Vorbereitung (Grüning, Schwerin), Durchführung (Heine, Halle) und Nachsorge (Weiß, Chemnitz) der internistischen Thorakoskopie sowie neue Entwicklungen und aktuelle Leistungsdaten (Hinrichs, Halle) vorgestellt.

Wichtige Schwerpunkte waren die Einordnung der Methode in die diagnostische Strategie des Pleuraergusses (Wülfing, Hemer) und der Stellenwert der aktuellen Pleurodeseverfahren mit besonderer Berücksichtigung der thorakoskopischen Talkumpoudrage (Stanzel, Hemer) sowie die Anwendung getunnelter Pleurakatheter (Hinrichs, Halle). Darüber hinaus wurden die Diagnostik und Therapie des komplizierten parapneumonischen Pleuraergusses und die Rolle der internistischen Thorakoskopie als eine wesentliche Behandlungsoption umfassend vorgestellt und diskutiert (Heine, Halle). Uta Wülfing (Hemer) berichtete über das diagnostische Management beim Verdacht auf das Vorliegen eines Pneumothorax, wobei die Rolle der Sonografie besondere Berücksichtigung fand. Der Stellenwert der internistischen Thorakoskopie als wesentlicher Bestandteil des therapeutischen Konzeptes (Talkumpoudrage) bei primären und sekundären Pneumothraces wurde von Froudarakis (Alexandroupolis) vorgestellt.

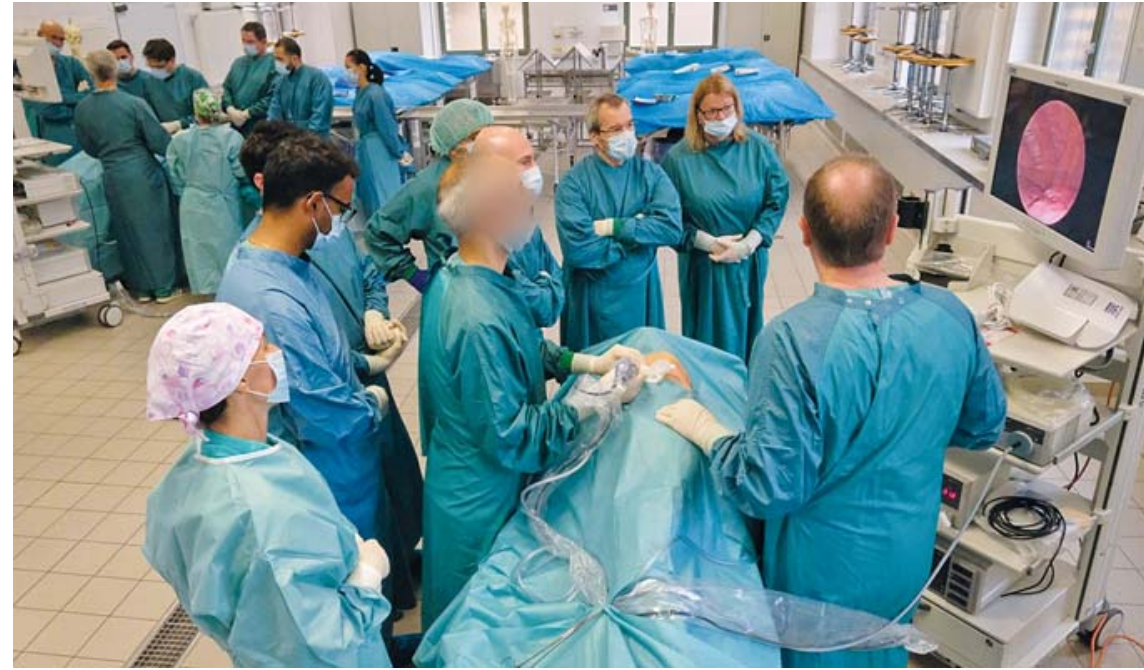

- Praktische Durchführung der Thorakoskopie am anatomischen Präparat (Anatomisches Institut der Martin-Luther-Universität Halle-Wittenberg). Bildquelle: Silvio Kison

Liedtke (Halle) gab eine Übersicht über Möglichkeiten, Probleme und Risiken der Analgosedierung im Rahmen der Durchführung der internistischen Thorakoskopie aus der Sicht des Anästhesiologen. Hajduch (Halle) berichtete über die aktuellen Möglichkeiten videoassistierter thoraxchirurgischer Operationsverfahren.

Neben Vorträgen zu anatomischen und pathophysiologischen Grundlagen (Frank, Berlin) und Fallvorstellungen (Spyratos, Thessaloniki (GR); Grüning, Schwerin) wurde die Veranstaltung durch Livedemonstrationen thorakoskopischer Eingriffe (Heine, Halle) bereichert. Neben der Pneumothoraxanlage demonstrierten die Untersucher anatomische Details, pathologische Pleuraveränderungen, Biopsietechniken aus der Pleura, die Talkumpoudrage, die Anlage herkömmlicher großlumiger Thoraxdrainagen und getunnelter Katheter.
Einen weiteren Schwerpunkt bildete ein Vortragsblock zu Themen der Hygiene (Pielert, Halle) und zu anderen Methoden der Diagnostik und Therapie von Erkrankungen der Pleura. Köpernick (Halle) gab eine umfassende Übersicht über radiologische Befunde. Frau Gütz (Leipzig) zeigte die Möglichkeiten der Pleuraergusszytologie auf. Außerdem gab es Übersichtsvorträge zur Pleurasonografie (Knappe, Leipzig), der Rolle der internistischen Thorakoskopie für die Diagnostik des Pleuramesothelioms (Stanzel, Hemer), die aktuelle Strategie der Mesotheliomtherapie (Busch, Halle).

Vorträge zu Indikationen und Techniken der Drainage der Pleura (Stanzel, Hemer), Problemen bei liegender Thoraxdrainage (Hinrichs, Halle) und bronchoskopischen Möglichkeiten der Behandlung persistierender Airlaeks (Heine, Halle) vervollständigten das Spektrum.

Ein Höhepunkt war der praktische Teil des Thorakoskopiekurses. Dieser fand erstmals in Kooperation mit dem Weiterbildungszentrum für Klinische Anatomie 
im Anatomischen Institut der Martin-Luther-Universität Halle-Wittenberg (Direktorin: Prof. Dr. Heike Kielstein) statt ( Abb.1). Unter Anleitung hatten alle Kursteilnehmer die Möglichkeit am anatomischen Präparat unter sehr praxisnahen Bedingungen die Methode selbst durchzuführen und anatomische Details kennen zu lernen.

\section{INFOKASTEN}

Der nächste Thorakoskopiekurs wird vom 19. bis 21. September 2019 in Halle/Saale stattfinden (begrenzte Teilnehmerzahl!!).

Anmeldungen:

Kerstin Marien/Susan Arlt

Medizinische Klinik III

Krankenhaus St. Elisabeth und

St. Barbara

Mauerstr. 5

06110 Halle/Saale

Tel.: 03452134281

k.marien@krankenhaus-halle-saale.de s.arlt@krankenhaus-halle-saale.de

Dr. Ralf Heine, Halle/Saale 